\title{
IMPLICATIONS FOR LEARNING A SECOND \\ LANGUAGE: GENERAL DISCUSSION
}

\author{
Moderator: Robbins Burling \\ Department of Anthropology \\ University of Michigan \\ Ann Arbor, Michigan 48109
}

Unidentified Speaker: How do you know that the Chinese tones weren't perceived as nonlinguistic units, such as musical tones, especially if the subjects did not know Chinese?

H. Winitz (University of Missouri, Kansas City, Mo.): I did speak to that issue in the paper, indicating that in Snow's paper the same general findings with regard to age and acquisition were obtained for linguistically meaningful elements. Furthermore, I referred to studies in which comprehension of language was taught and in which adult subjects performed at a high level, as did the adults in my study. Therefore, I believe that the listening capabilities of adults are generally superior to children in the early stage of language acquisition. Although the discriminations I was testing may not be regarded as linguistic events, it is of interest to observe that they are skills that are essential if oral language is to be acquired, and in that regard, adults seem initially to have more advanced abilities than children have.

C. Yorio (University of Toronto, Toronto, Ontario, Canada): Dr. Belasco, many of the examples that we have heard are fairly traditional, in the sense that you are presenting what one could call a structural syllabus. The point is, what is your feeling about the application of this approach to a functional syllabus?

S. Belasco (University of South Carolina, Columbia, S.C.): My remarks pertain to the first semester only. I outlined what the students are able to do in the first semester. Teaching speech acts, illocutionary acts, takes place in the second semester. In the first semester, the emphasis is placed on interpretation and not on production. The goal of these procedures is to improve comprehension and to reinforce comprehension.

The purpose of the translation exercises is to teach the student to concentrate on the perception and interpretation of utterances. Exposure to "illocutionary acts" in the form of readings and comprehension exercises must precede the actual teaching of the use of illocutionary acts in conversational situations.

C. YoRIo: One more quick question about correction and accuracy. You talked in particular about stopping the student when he makes a mistake. If your concern is with comprehension, during the first semester, why stop him if he has communicated? Finally, when you have a trans- 
lation test for them to write out, do you correct for errors in spelling, accent marks, etc.?

S. Belasco: What I want the student to do is approximate the pronunciation. I am interested in his knowing sandhi variation, when to make liaison, when not to make liaison, about dropping final consonants, and so forth. If the student doesn't repeat correctly, I call on another student. Most importantly, content is stressed. Later I correct for errors.

R. BlaKe (Dartmouth College, Hanover, N.H.): Dr. Asher, my question relates to your remarks regarding caretakers. Not all caretakers provide a warm and supportive environment for the child. Children who come from the most abusive and deprived home conditions learn to talk. I would like to hear you comment about that.

J. J. Asher (San José State University, San Jose, Calif.): The clinical literature suggests that there is a relationship between the quality of caretaking and impairments in child development. For example, neglect of infants can slow the rate of language acquisition, and even produce aberrations in understanding and production.

Unidentified SPEAKER: Dr. Asher, how does your total-physicalresponse approach compare with other ways of achieving comprehension?

J. J. ASHER: Most approaches for comprehension training, such as the ones developed by Asher, Winitz, Nord, the Garys, Postovsky, and others, are in harmony because they play to the right hemisphere of the brainwhich is the kind of stimulation that infants experience especially in the pretalking period of language development.

Unidentified SPEAKER: I have a question, Dr. Gary. After some 80 hours of training, what can we expect from the students? Can they write or answer in English? And furthermore, how many lexical items do they know?

N. GARY (English Language Center, Cairo University, Cairo, Egypt): At the end of the 80 hours of instruction, the students average $85 \%$ proficiency in comprehension and reading on the structures they have been studying. The tests are not simple repetitions of items. They include new combinations. We do not emphasize writing, and so we do get terrible spelling. However, the students are able to write out appropriate messages in accord with their level of training. Speaking could not be fairly assessed given the constraints of our program. However, speaking has been assessed under controlled conditions in other studies. Finally, the students learn 1,000 to 1,200 lexical items during this period of instruction. 